bacteriological reports their true value is worthy of the most careful consideration. It is well recognised that of all the microscopic forms of life found in so many samples of water, only the most infinitesimal proportion has any casual relation to disease, and some investigators have gone so far as to say that the free growth of ordinary water organisms forms one of our best protections against the development of pathogenic forms, so that in a multitude of microbes we have safety. It is easy then to see that it is far more important to determine the kind than the number of the organisms present. But the hunt for pathogenic forms has not been very successful. Water which has been known to be dangerous has so often failed to show the presence of disease germs that no bacteriologist would nowadays dare to pronounce as to the innocuous nature of any sample on the ground of its appearing free from recognisable pathogenic forms. Thus the bacteriologist has been reduced to much the same position as the chemist, and although it may happen, now and then, that he may feel justified in asserting that a given sample is infected with a given germ, in the vast majority of instances he has to be content with saying that it shows evidences of previous sewage contamination; these evidences consisting generally of the presence of more than a minimal proportion of certain organisms which are known to be chiefly if not exclusively produced in the intestinal canal. Unfortunately neither the chemist nor the bacteriologist have hitherto been able to say with any great certainty how recent is this "previous sewage contamination." But this is often the very crux of the whole question, for pathogenic organisms are in many cases short-lived, and the danger of the contamination depends largely upon the date at which it took place. Under these circumstances great interest attaches to the researches made by Dr. Houston as to the significance of the presence of streptococci in water, detailed in the recently-issued annual report of the Medical Officer to the Local Government Board ; for if, as he shows, these organisms can only maintain their independent existence in water for a short period, their presence may be taken as proof of very recent contamination. The two organisms on the presence of which dependence is mostly placed as proving previous sewage contamination are the B. coli and the B. enteritides sporogenes. The latter, however, being a spore-bearing organism, its presence cannot be taken as evidence of necessarily recent pollution; and, although it may fairly be said that the presence of $\mathrm{B}$. coli in any number in either soil or water implies recent pollution of animal sort, the word " recent" in this regard must be taken as having a somewhat wide meaning. But, says Dr. Houston, is there any microbe or class of microbes which by its presence implies animal pollution of extremely recent, and therefore specially dangerous kind? And he answers that the streptococci are to be thought of in this sense. The position may be put thus: on the one hand in pure water and in the washings of virgin soil he has uniformly failed to detect microbes of the streptococcus class, while on the other hand streptococci are habitually found in such small volumes of crude sewage as one-thousandth part of a cubic centimetre, and are ordinarily abundant in minimal quantities of human excrement. Thus we are led to the belief that the discovery of streptococci in a water sample affords a very valuable indication, not merely that the water has been contaminated by animal organic matter, but that the contamination in question has been of quite recent occurrence and thus of a sort peculiarly dangerous to a water service.

If further experience should show that this test is to be relied upon as an indication of the date of a contamination, and if we are thus enabled to say, not merely that a given sample of water has some time or other been exposed to sewage contamination, but that this contamination has so recently left the human body that if it contained pathogenic organisms these would probably be alive and in full activity, then a great advance will have been made in the position occupied by bacteriology in water examination.

\section{A PHARYNGEAL POUCH.}

At the last meeting of the Royal Medical and Chirurgical Society a case was related by Messrs. Rickman Godlee and Bucknall, in which a large pharyngeal pouch was removed by operation. On coming under observation in May, 1900, the patient had a swelling which looked exactly like an enlarged gland on the left side of the neck, at the level of the hyoid bone, in front of the sternomastoid. It was, however, soft; on percussion it gave a tympanitic percussion note, and it was clearly anchored to the top of the larynx, the movements of which it followed during deglutition. Pressure on the tumour led to an obvious diminution in its size, produced a squeaking sound, and at the same time caused pain extending to the ear which persisted for some days. Removal of the pouch was advised, but the patient could not make up his mind and he. was not seen again until December 31, when he was admitted to University College Hospital. The history he gave was interesting. He was 31 years of age. Twelve years ago he had received a blow from a fist on the left side of the neck, which, although painful, was followed by no special symptoms at the time. Two years after this he began to feel something working up and down on the left side of the neck on swallowing, and during the last three years he had been liable to acute attacks, lasting usually about a fortnight, during which a lump developed on the left side of the neck, and swallowing became increasingly difficult and painful until he could only swallow fluids. The last attack had been much worse than usual, and for the first time the skin over the swelling had become red. On admission the tumour was very painful, and reached the middle line in front, and the posterior border of the sterno-mastoid behind, overlapping and pressing back this muscle. The tumour was freely movable over the deeper parts except near the upper part of the larynx to which it was fixed, the carotid vessels passed behind it. The percussion note all over the tumour was tympanitic, and he could slightly inflate it by holding his breath and blowing. The pouch was removed on January 2. The dissection required was rat her a delicate matter. The upper part of the pouch was found to consist of two portions communicating by a rather small opening, but when the narrow pedicle was reached, which passed through the thyro-hyoid membrane, careful probing failed to discover the actual communication with the pharynx. The tubular pedicle was secured by catgut stitches, a portion of it being divided as each stitch was passed, and finally the stump was invaginated by four stitches passed after the manner of Lembert's sutures. The wound healed perfectly. About 200 cases of pharyngeal pouch and fistula have been recorded. They probably all arise in connection with one or other of the visceral clefts, and the following varieties were given by the 
authors of this paper as depending upon the position of the obliteration of the cleft : (1) it may remain open throughout its entire length, giving rise to the complete fistula opening into the pharynx and on the side of the neck; (2) no external opening may be formed and a pharyngeal pouch with a blind extremity may remain; (3) the internal aperture may become shut off from the pharynx, leaving a blind external.fistula; and (4) both internal and external openings being closed a "dermoid" cyst of the neck may remain. In more than a third of all the cases the fistula was complete, and of the remainder the majority presented an external fistula, either blind or with the internal orifice so concealed that it could not be found. It is at least probable that some of the so-called pressure pouches of the œsophagus originate from pouches which may be placed in the same category as the above.

\section{THE PULSE IN PERITONITIS.}

Again and again it has been pointed out that the pulse is the great guide when the peritoneum is involved, and that, notwithstanding the favourable character of other symptoms, if the pulse remains quick we have no right to be satisfied with the condition. The converse, however, may not hold true. A case recorded by Bowlby and Steedman ${ }^{1}$ serves well to show how careful we must be not to place reliance upon single symptoms; for here, although the abdominal cavity contained about a quart of darkly blood-stained fluid, and the greater part of the small intestine was so twisted as to form a large volvulus, the pulse remained below 100 in spite of the strangulation of so large a mass of intestine, and although the patient was evidently much collapsed and in intense pain. The case was a very remarkable one in other respects, this volvulus having come on after a previous operation for perforated gastric ulcer in which rigors had occurred; but the authors draw special attention to this question of pulse-rate, saying: "It is seldom that so serious a condition as was found in this case on opening the abdomen causes so little disturbance of the pulse-rate; but this case, and others which we have seen, should serve as a warning not to place too much reliance on a quiet pulse when the question of laparotomy is under consideration for the relief of such suspected conditions as perforated gastric ulcer and volvulus."

2 Lancet, May. 4.

\section{A HAT-PIN SWALLOWED BY AN INFANT.}

A curious case is reported from St. Thomas's Hospital under the care of $\mathrm{Mr}$. Ballance. ${ }^{1}$ The patient was an infant aged 15 months, who, 18 hours before admission, while standing on a chair had placed a lady's hat-pin (six inches long) headforemost in his mouth. He fell from the chair, and the pin, probably from striking the ground, was pushed down his throat. His mother could see the point, but in trying to pull it out only pushed it further down. The child subsequently swallowed two pieces of toast without any difficulty, and when brought to the hospital showed no signs of anything being amiss. Skiagrams, however, proved the pin to be in the lower end of the osophagus, the head being about the level of the cardiac end of the stomach. Thirty hours after the accident the stomach was opened, the cesophagus having first been explored by the finger without result. The stomach was exposed and held up to the surface by two threads passed into its wall, and a small incision was made between the threads. On the finger being introduced the head of the pin was felt presenting at the cardiac opening, and it was extracted without difficulty. For 24 hours the child was fed per rectum. He recovered perfectly. Some time ago a case was recorded in which a similar accident happened to an adult. A woman, thinking that something she was eating had stuck in her throat, tried to ram it down with her hat-pin. In so doing the point got jammed behind her upper incisor teeth, and while she was endeavouring to dislodge it the pin slipped from her fingers and was swallowed. In this case the whole of the pin entered into the stomach and at the operation the surgeon, instead of incising the stomach wall, pushed the point through until the head prevented its further passage and then, cutting the pin off short with wire nippers, left the head to pass away per vias naturales, which it did.

$$
{ }^{1} \text { Clinical Journal, May } 15 .
$$

\section{VARICELLA IN ADULTS.}

According to Dr. Doty, health officer to the Port of New York, a widespread belief exists that varicella does not affect adults, but is confined to infancy and childhood. This, however, is not true, and it is fully understood by those familiar with these diseases that chicken-pox may occur after puberty. The diagnosis between small-pox and chicken-pox depends, he says, upon (1) the character of the eruption, (2) the manner in which it appears, and (3) its distribution or location. In regard to the manner in which it appears in small-pox, the eruption presents itself in one crop, and passes through its different stages practically together, as papules, vesicles, and pustules. In some parts of the body the formation of each stage, or the transition from one stage to the other, may be a little more rapid than others; but when this is finished the eruption is complete, and secundary crops never appear. In chicken-pox, on the other hand, the eruption comes out in a manner diametrically the opposite of this. Alongside of vesicles which have dried down with the formation of a black or dark scab we find small tender vesicles just appearing, which are torn by a slight touch of the nail. Moreover the eruption of chicken-pox appears abruptly as vesicles, and is not preceded by papules as in small-pox. As to the distribution of the eruption, in small-pox, even in mild cases, the hands and feet are to some extent almost always involved; whereas in chickenpox, even with a profuse eruption, the hands and feet are either unaffected or have but little eruption. The appearance of hard, tough, circumscribed, and distended papules or vesicles on the hands or feet, particularly on the palms and soles, is an exceedingly important diagnostic sign of smallpox. Even in mild cases we are quite sure to find a few along the fingers and toes and palms and soles, while in varicella the hands and feet are singularly free, even when the eruption is profuse on other parts of the body. It may be added that the back presents the best surface on which to study the eruption of varicella. As to umbilication this is a well marked and valuable diagnostic sign in small-pox, but what will pass for and is often regarded as umbilication is frequently found in chicken-pox as well as in some forms of syphilitic eruption. Under all circumstances it should be borne in mind that the diagnosis of small-pox and chicken-pox is made from the appearance of the eruption. The constitutional symptoms are important as corroborative evidence, but they should be considered after the eruption has been studied. The more proficient one becomes in the diagnosis of small-pox or chicken-pox the less he relies on constitutional symptoms. 